\title{
Do Kuhn's Cases of the Theory-Change from Newtonian to Einsteinian Physics Support His Incommensurability Thesis?
}

\author{
Kyoung-Eun Yang*
}

Received: 28 April 2020 / Revised: 22 September 2020 / Accepted: 8 October 2020

Abstract: In order to support his revolutionary view on scientific change, Kuhn suggests that there exist two separate aspects of the theory-change from Newtonian to Einsteinian physics that support his incommensurability thesis. For the evidence of his thesis Kuhn offers the conceptual change in the meaning of notion of "mass" in the theory-change. And he claims the absence of any neutral observational basis to evaluate the strengths of the two theories. This essay argues that these two cases fail to support his incommensurability thesis.

Keywords: Conceptual change of mass; Kuhn's incommensurability thesis; neutral observational basis; scientific revolution; space-time measurement; the theory-change from Newtonian to Einsteinian physics.

* Korea National University of Education

(D) https://orcid.org/0000-0001-8967-6127

- Graduate School, Korea National University of Education, 250 Taeseongtabyeonro, Gangnae-myeon, Heungdeok-gu, Cheongju-si, Chungcheongbuk-do, South Korea

$\bowtie$ newtleib@gmail.com

(C) The Author. Journal compilation (c) The Editorial Board, Organon F.

This article is distributed under the terms of the Creative Commons Attribution-NonCommercial 4.0 International Public License (CC BY-NC 4.0). 


\section{Introduction}

The theory-change from Newtonian to Einsteinian physics has been regarded as an archetypal example of a revolution in science by both scientists and philosophers. For example, the physicist Max Born identified the development of the theories of relativity as "the Einsteinian revolution" which opened "the beginning of a new era" (Born 1965, 2). And the philosopher of science Karl Popper wrote that "Einstein revolutionized physics" (Withrow 1967, 25). After the prediction made by general relativity of the deflection of light due to the sun's gravitational field was confirmed by observations, the London Times on November $7^{\text {th }} 1919$ famously ran the headline "Revolution in Science" with a sub-heading "Newtonian Ideas Overthrown." In view of the fact that Einsteinian physics is founded on his novel understanding of the concepts of space-time and of the equivalence of mass and energy, which are quite alien to the Newtonian frameworks, it seems difficult to deny the intuition behind the idea that this episode of theory-change was indeed revolutionary.

Along these lines, Kuhn (1962) explicitly articulated and defended a revolutionary view involving "paradigm changes." According to Kuhn, two different paradigms are incommensurable in their assertions about the world, aims, criteria of appraisal, conceptual frameworks, and even observational basis. As supporting cases, Kuhn considers the conceptual discontinuities concerning notions such as 'mass' and 'space-time' as evidence of the occurrence of a revolution brought about by Einsteinian physics.

Einstein himself, however, rarely employed the term "revolution" in order to characterize his theories of relativity (Cohen 1985). He instead warned that the term "revolution" mischaracterizes the way that the special and the general theories were developed. Their development is considered as one which "slowly leads to a deeper conception of the laws of nature" based on results of "the best brains of successive generations" (Klein 1975, 113). Although Einstein referred to the theory-change from Newton to Maxwell as a revolution in that "action at a distance is replaced by the field" (Einstein 1949, 35), he did not maintain that the theories of relativity were new fundamental theories. The special theory of relativity is claimed as "simply a systematic development of the electromagnetics of Maxwell and 
Lorentz" (Einstein 1934, 57). As for the general theory it was "the last step in the development of the program of field theory, ... [and] it modified Newton's theory only slightly" (ibid., 37).

This paper follows Einstein's intuition - that the development of his two theories of relativity is not in fact revolutionary - in critically examining the aforementioned Kuhn's two cases in the theory-change from Newtonian to Einsteinian physics. This essay will argue that these two cases fail to support the incommensurability thesis. ${ }^{1}$

\section{Kuhn's scientific revolution and conceptual changes in Einsteinian physics}

Thomas Kuhn famously argued in his Structure of Scientific Revolutions that there have been major discontinuous changes in the history of science. He explicitly cites the theory-change from Newtonian to Einsteinian physics as a case strongly supporting his claim (Kuhn 1962).

The concept of paradigm in Kuhn's view plays a key role in characterizing the different stages of science. In the stable stage during which a specific scientific discipline matures, the discipline, which Kuhn calls a 'normal science,' "is predicated on the assumption that the scientific community knows what the world is like" (Kuhn 1962, 5). Scientists make great efforts to preserve this assumption, to the extent that "normal science often suppresses fundamental novelties because they are necessarily subversive of its basic commitments" (ibid.). Research within normal science is thus based on a 'paradigm,' which consists of the scientific community's metaphysics, conceptual frameworks, theories, methodology, and goals. A paradigm is essential to normal science, in that "no natural history can be interpreted in the absence of at least some implicit body of intertwined theoretical and methodological belief" (ibid., 16-7). Adopting a paradigm is "an attempt to force nature into the preformed and relatively inflexible [conceptual] box that the paradigm supplies" (ibid., 24). So, "[n]ormal-scientific research is

1 Surely, I cannot dismiss Khun's general argument just because his specific example is dubious. This paper targets only Kuhn's argument that is based on the the theory-change from Newtonian to Einsteinian physics.

Organon F 28 (2) 2021: 458-483 
directed to the articulation of those phenomena and theories that the paradigm already supplies" (ibid.).

However, with the advent of a crisis within the paradigm (if only in some vague sense) - caused by the continuing failure to solve anomalies, the paradigm confronts challenges from competitors that question the fundamental assumptions underlying the earlier normal science: "[N]ature has somehow violated the paradigm-induced expectations that govern normal science. It then continues with a more or less extended exploration of the area of anomaly" (ibid. 52-3). And the "crisis may end with the emergence of a new candidate for paradigm and with the ensuing battle over its acceptance" (ibid. 84).

One of the competing paradigms, because of its success in solving the anomalies, attracts advocates who set the direction of their future research according to the new paradigm. "The transition from a paradigm in crisis to a new one from which a new tradition of normal science can emerge is far from a cumulative process ... Rather it is a reconstruction of field from new fundamentals, a reconstruction that changes some of the field's most elementary theoretical generalizations" (ibid. 84-5). A scientific revolution occurs when the new paradigm replaces the old one. One result is that different paradigms are "incommensurable" in their aims, conceptual frameworks, and even observational bases: "The normal-scientific tradition that emerges from a scientific revolution is not only incompatible but often actually incommensurable with that which has gone before" (ibid. 103). Kuhn's incommensurability thesis involves several radical claims - for example that even the empirical data for a given theory cannot be translated in a way that is neutral between competing paradigms:

Scientists then often speak of the "scales falling from the eyes" or of the "lightning flash" that "inundates" a previously obscure puzzle, enabling its components to be seen in a new way that for the first time permits its solution. ... No ordinary sense of the term 'interpretation' fits these flashes of intuition through which a new paradigm is born. Though such intuitions depend upon the experience, both anomalous and congruent, gained with the old paradigm, they are not logically or piecemeal linked to particular items of that experience as an interpretation would be. Instead, 
they gather up large portions of that experience and transform them to the rather different bundle of experience that will therefore be linked piecemeal to the new paradigm but not to the old. (ibid., 122-23)

In order to support his revolutionary view, Kuhn suggests that there were two separate aspects of the theory-change from Newtonian to Einsteinian physics that support the incommensurability thesis. These are the conceptual change in the meaning of notion of "mass" (ibid., 102), and the absence of any neutral observational basis to evaluate the strengths of the two theories due to the "theory ladenness" of the space-time measurements in the two theories (ibid., 149-50).

In the following sections, I will argue that these two claims fail to support the incommensurability thesis.

\section{The case of the concept of mass}

According to Kuhn, although the terms employed in Newtonian physics such as mass are also employed in Einsteinian physics, the referents of these terms are not the same:

[T]he physical referents of these Einsteinian concepts [mass] are by no means identical with those of the Newtonian concepts that bear the same name. ... Only at low relative velocities may the two be measured in the same way, and even then they must not be conceived to be the same. (Kuhn 1962, 102)

In the same spirit, Paul Feyerabend pointed out:

[I]n classical, prerelativistic physics the concept of mass (and, for that matter, the concept of length and the concept of time duration) was absolute in the sense that the mass of a system was not influenced (except, perhaps, causally) by its motion in the coordinate system chosen. Within relativity, however, mass has become a relational concept whose specification is incomplete without indication of the coordinate system to which the spatio-temporal descriptions are all to be referred. ... what is measured in the classical case is an intrinsic property of the system under 
consideration; what is measured in the case of relativity is a relation between the system and certain characteristics of [the coordinate system] D'. (Feyerabend 1962, 80)

Although the term 'mass' appears in both theoretical frameworks, the Newtonian mass of a body is the same irrespective of its state of motion, whereas the Einsteinian mass varies depending on the motion of a body relative to the frame within which its mass is measured:

[T] he total mass of a system is not a scalar quantity in relativity theory, so that its value depends on the reference frame with respect to which it is measured. For example a particle whose mass is $m$, as measured in its own rest frame, appears to have a larger mass when measured in a second frame with respect to which it is moving. (Penrose 2004, 435)

In other words, the Newtonian mass is a scalar quantity m, which is invariant under any coordinate transformation, while the Einsteinian mass (expressed as $\mathrm{M}=\gamma \mathrm{m}$, where $\gamma$ is the Lorentz factor, i.e., $1 / \sqrt{ }\left(1-\mathrm{v}^{2} / \mathrm{c}^{2}\right)$ ) is a variable quantity which increases with the velocity $\mathrm{v}$ of the body.

Feyerabend claims that the change in the concept of mass shows that there are enormous difficulties in relating the two successive scientific theories:

It is also impossible to define the exact classical concepts in relativistic terms or to relate them with the help of an empirical generalization. ... It is therefore again necessary to abandon completely the classical concepual scheme once the theory of relativity has been introduced ... Our argument against meaning invariance is simple and clear. It proceeds from the fact that usually some of the principles involved in the determination of the meanings of older theories or points of view are inconsistent with the new, and better, theories. (Feyerabend 1962, 80-2)

The concepts of classical and relativistic mass essentially belong in "different and incommensurable frameworks" (ibid., 81). In his recent book on concepts of mass, Jammer describes the relationship between the concepts of invariant and relativistic masses as "ultimately the disparity between two competing views of the development of physical science" (Jammer 2000, 
61). Kuhn also sees this conceptual change as a classic illustration of the incommensurability thesis:

the normal-scientific tradition that emerges from a scientific revolution is not only incompatible but often actually incommensurable with that which has gone before. (Kuhn 1962, 103)

This case, however, fails to provide legitimate evidence supporting the occurrence of a radical conceptual change. Einsteinian relativistic mass cannot in fact be considered as a physically significant concept that is the counterpart of the Newtonian mass. In fact, the former is not a legitimate physical quantity that respects the principles of special relativity. Kuhn explicitly takes the 'relativistic mass' $M=\gamma \mathrm{m}$, which increases with the velocity of the body. Yet this concept of mass does not properly fit within the framework of special relativity.

The special theory of relativity is essentially based on two fundamental postulates: (1) all physical laws take the same form in all inertial frames, and (2) the speed of light is always the constant $\mathrm{c}$ in all such frames. From these two hypotheses, Einstein derived the coordinate transformation which implements the principle of special relativity. ${ }^{2}$ As a result, these two hypotheses yield predictions about the kinematical effects of time dilation, length contraction, and the addition of velocities. On the basis of this kinematics, a dynamical framework can be developed by positing the concepts of mass and momentum. In this context, as the above expression suggests, one can employ the relativistic concept of mass $\mathrm{M}_{\mathrm{rel}}$ while maintaining the classical concept of velocity $\mathrm{V}_{\mathrm{cla}}(\mathrm{dx} / \mathrm{dt})$ in order to relativitize the concept of momentum, i.e. prel $=\mathrm{M}_{\text {rel }}$ cla. In other words, the Newtonian momentum $\left(\mathrm{p}_{\mathrm{cla}}=\mathrm{m}_{\mathrm{cla}} \mathrm{V}_{\mathrm{cla}}\right)$ is modified into the relativistic expression by adopting the relativistic mass (rather than by changing laboratory time dt to proper time $\mathrm{d} \tau)$, i.e., $\gamma \mathrm{mdx} / \mathrm{dt}$. At this point, however, one is forced to adopt a primitive concept of improper 4-velocity, which is not Lorentz covariant. (Oas 2006, 4) "The improper velocity being a direct result of the imposition of RM [the relativistic mass] means that RM is at odds with the accepted kinematics of special relativity" (ibid.).

2 Accordingly, one of essences of the special theory of relativity is that the laws of physics must be covariant with respect to Lorentz transformations.

Organon F 28 (2) 2021: 458-483 
In a similar spirit, Wheeler and Taylor claim that:

Any difference between [relativistic] formulae for momentum (for example, $\mathrm{mdx} / \mathrm{d} \tau)$ and the corresponding Newtonian formula $(\mathrm{mdx} / \mathrm{dt})$ is therefore to be attributed to the difference between proper time and laboratory time, not to any difference in the value of $\mathrm{m}$ in the two descriptions of nature. (Wheeler and Taylor 1963, 108)

Given that the Lorentz factor $1 / \sqrt{ }\left(1-\mathrm{v}^{2} / \mathrm{c}^{2}\right)$ measures the ratio between laboratory time and proper time, modifying the kinematical concept of velocity $\mathrm{dt}$ to $\mathrm{d} \tau$ is more natural than modifying the concept of mass. So, in order to be consistent with the kinematics of special relativity, the Lorentz factor $1 / \sqrt{ }\left(1-\mathrm{v}^{2} / \mathrm{c}^{2}\right)$ needs be associated with velocity, rather than with mass. This is expressed by Resnik as follows:

Indeed, it should be noted that, whether we identify the factor $1 / \sqrt{ }\left(1-\mathrm{v}^{2} / \mathrm{c}^{2}\right)$ with mass or with velocity, the origin of this factor in collision measurements is kinematical; that is, it is caused by the relativity of time measurement. (Resnik 1968, 199)

Because of this, Einstein himself considered the rest mass $m$ as the only physically significant concept and substituted the energy-momentum 4-vector for the relativistic mass. Thus, he wrote:

It is not good to introduce the concept of the mass $\mathrm{M}=\mathrm{m} / \sqrt{ }(1-$ $\mathrm{v}^{2} / \mathrm{c}^{2}$ ) of a body for which no clear definition can be given. It is better to introduce no other mass than 'the rest mass' $\mathrm{m}$. Instead of introducing $\mathrm{M}$, it is better to mention the expression for the momentum and energy of a body in motion. (Einstein 1948, a letter to Lincoln Barnett, quoted from Okun 1989, p. 42)

In the special theory, just as space and time are incorporated within the single entity space-time whose components are $(t, x)$, so energy and momentum are united to form the energy-momentum 4-vector whose components are $(\mathrm{E},-\mathrm{p})$, where we use $\mathrm{c}=1$ convention. This quantity satisfies a conservation law, which, given the equivalence of energy and mass, incorporates the law of mass conservation, the law of energy conservation, and the law of momentum conservation. The squared magnitude of this four- 
vector represents the rest mass, $\mathrm{m}^{2}=\mathrm{E}^{2}-\mathrm{p}^{2}$, which is invariant regardless of the choice of inertial frames. In this context, 'relativistic mass' is only the temporal component of the energy-momentum 4-vector of a given body. This single component is measured as being larger in motion than when the body concerned is at rest. However, all four components of the energy-momentum 4-vector are transformed in the proper way to maintain the vector's invariance under the change of the reference frame. Along these lines, Wheeler and Taylor's answer to the question "is the mass of a moving object greater than the mass of the same object at rest?" is "no":

The concept of 'relativistic mass' is subject to misunderstanding. That's why we don't use it. First, it applies the name massbelonging to the magnitude of a four-vector - to a very different concept, the time component of a four-vector. Second, it makes increase of energy of an object with velocity or momentum appear to be connected with some change in internal structure of the object. In reality, the increase of energy with velocity originates not in the object but in the [kinematical] properties of space-time itself. (Taylor and Wheeler 1992, 250-51)

It seems that Kuhn's reading of the concept of mass is mistaken.

Defenders of Kuhn's thesis that radical change is involved in this shift could respond that although Kuhn may have misunderstood the way in which the concept of mass has been radically transformed, adopting the concept of energy momentum still shows that the concept of "mass" undergoes a radical change. For example, the difference between the classical notion of mass and the energy momentum 4-vector is very apparent. Contrasted with classical mass, the components of the energy-momentum 4vector are described differently with the change of the coordinate systems representing inertially moving observers. Moreover, unlike classical mass, its relativistic counterpart is implicated in the interactions among mass, energy, and momentum. This shows the ontological difference between the notions of classical mass and energy-momentum. Accordingly, it could be argued that my argument resurrects Kuhn's view by introducing the concept of the energy-momentum 4-vector.

To answer this criticism, I will analyze the reference and denotation of the theoretical terms, i.e., classical mass and energy-momentum 4-vector. 
Bird (2000) and Field (1973) characterize the comparison of theoretical terms in theory-change as a matter of denotating and extending them. Consider Kuhn's claim "the physical referents of these Einsteinian concepts are by no means identical with those of the Newtonian concepts that bear the same name" (Kuhn 1962, 101). In response to this Kuhn's claim, Bird maintains that

Kuhn assumes that laws fix intensional meaning and thereby also fix, in a strict manner, extension and reference. If it is further assumed that all laws play a part-thick intensionalism-then it follows that if the term in question refers, then the laws must be true; correspondingly if one or more of the laws fails to be true then there is no reference, in which case incommensurability of reference cannot arise. (Bird 2000, 176)

In response to this claim by Kuhn, Bird maintains that Newtonian mass does not refer to anything since Newtonian law is not true. Along a similar line, Field points out that "what Einstein showed is that there $i s$ no such quantity as "Newtonian mass"; and unless one holds that the world used to obey Newton's laws but started obeying Einstein's laws one day, it is clear that there was no "Newtonian mass" in Newton's time either" (Field 1973, 470).

However, physicists would disagree about this skeptical attitude toward Newtonian mechanics. Field also endorses the physicists' intuition since, to a certain extent, the reference of Newtonian mass is based on "a great many false-but-approximately-true beliefs" (ibid., 465). Accordingly, Field modifies Frege's descriptivist theory and embraces the idea that there can be the partial denotation of scientific terms such as Newtonian mass. Along the similar line, Bird claims that

it need not be that the total content of a given law contributes to meaning, and indeed it is implausible that it should be so. But then the content of laws breaks up into an element that determines reference and an element that does not. (Bird 2000, 176)

In order to clarify the reference-determining elements of Newtonian mass, Field directs our attention to the theoretical terms of Einstein's special relativity, which converge on Newtonian mass. Given that "at low 
velocities, the mass of a particle is almost precisely equal to twice its kinetic energy divided by the square of its velocity," this concept refers to either relativistic or proper mass (Field 1973, 464-5).

What is notable about Field's account is that the concept of the "falsebut-approximately-true" Newtonian mass is referentially indeterminate (ibid., 467). The central idea of Newtonian mass can be captured by (1) momentum $=$ mass $\cdot$ velocity, and (2) the quantity of mass is the same regardless of the choice of reference frame. However, (1) and (2) cannot both be true given that special relativity does not authorize the conjunction of (1) and (2). Therefore, it seems possible to specify the one that is not correct. However, Field claims that there are contexts in which physicists legitimately employ both the relativistic mass and the proper mass by endorsing only one of the two theoretical tenets. For example, they might suggest that momentum equals the relativistic mass times velocity, but that it by no means equals the proper mass times velocity. By contrast, the proper mass is the same regardless of the choice of reference frames, whereas the relativistic mass is not. According to Field,

The conjunction of Newton's tenets (1) and (2) was objectively false, but there is no fact of the matter as to which of the conjuncts was true and which false, and hence no fact of the matter as to whether the word 'mass' as it occurred in them denoted relativistic mass or proper mass. (ibid., 468)

Newtonian mass can be approximated through the Newtonian limit of either the relativistic mass or the proper mass. However, "there is no fact of matter as to how the falsity of the theory as a whole is to be distributed among the individual sentences of the theory" (ibid., 474). Hence, there is no fact of the matter in theoretical terms as to what the approximate truth ascribes. Accordingly,

there is simply no coherent way of using the term 'refers' in connection with Newton's word 'mass'. In spite of this, there are many of Newton's utterances containing the word 'mass' that we want to regard as true ... It follows, then, that the truth and falsity of these utterances simply cannot be explained on the basis of what Newton was referring to when he used the word 'mass', 
for there is no coherent way of explaining what he was referring to. (ibid., 473)

Kuhn claims the incommensurability thesis since the term "Newtonian mass" refers to an invariant quantity, whereas the term "Einsteinian mass" does not. In opposition to Kuhn's claim that the referents of these two concepts are different, Field argues that these two concepts can both be the referents for the term "Newtonian mass." According to Field, the physical referent of both Newtonian mass and Einsteinian mass is either the relativistic mass or the proper mass. He maintains that the referentially indeterminate Newtonian mass and Einsteinian mass can refer to both, and he makes this claim while criticizing Frege's referential semantics.

I will modify Field's argument in line with my employment of the energy-momentum 4-vector. Field argues for referential indeterminacy since Newtonian mass can refer to both the relativistic and the proper mass. This is because physicists can legitimately employ both theoretical terms. However, as I have argued before, physicists prefer the proper energy momentum 4-vector to the improper relativistic mass. Nevertheless, I still endorse Field's notion of referential indeterminacy, even if I come at it from a different point of view. This is because the energy-momentum 4-vector unifies both the relativistic mass and the proper mass.

Einstein (1905) constructed the special theory of relativity in his attempt to incorporate the electric field and the magnetic fields, and correspondingly space and time: "The existence of the electric field [and the magnetic field] was therefore a relative one, dependent on the coordinate system used, and only the electric and magnetic field taken together could be ascribed some kind of objective reality" (Einstein 1919). As a result, mass, energy, and momentum are all incorporated into the energy-momentum 4-vector. Thus, the energy-momentum 4-vector contains both referential elements for the relativistic and proper mass. The temporal aspect of the energy-momentum 4-vector represents the relativistic mass, while its all four components together represent the proper mass. The former is true for one observer, while the latter is true for another observer. This would be a plural denotation on top of an indeterminate one.

Morrison considers this case of unity as a synthetic one that "involves the integration of two separate processes or phenomena" under one concept. 
In this case, there is no ontological reduction that "offers little in the way of support for claims about a physical unity in nature" (Morrison 2000, 5). For Field, the physical referent of both Newtonian mass and Einsteinian mass is either the relativistic mass or the proper mass. By contrast, I argue that the physical referent of both kinds of masses is the energy-momentum 4 -vector, which incorporates both the relativistic mass and the proper mass. By taking this slightly different route, I arrive at the same conclusion as Field's one. Along the similar line, Bird maintains that

It could be that the reference-determining element is sufficiently general as to be applicable to E-mass as well as N-mass, in which case those will be the same property. Thus there will be no incommensurability of reference in this case... Consequently his argument fails to establish incommensurability due to shifts in reference. (Bird 2000, 176)

Accordingly, Kuhn's incommensurability thesis could be undermined if one considered the energy-momentum 4-vector as this reference-determining element.

The reference-determining elements of scientific theories that undergo theory-change are best captured by the so-called convergent realists. Hardin and Rosenberg (1982) claim that although we can admit that central theoretical entities in theories from the past do not refer to their intended objects, we can still talk about approximate truth due to the commonality between the past and present-day theories about the same causal or explanatory role. Kitcher's distinction between "working posits" and "presuppositional posits" is based essentially on whether theoretical terms are referring or non-referring (Kitcher 1995).

At this point, it seems appropriate to add Duhem and Poincaré's idea into our context, given that they were concerned with the theory-change related to mechanics (Duhem 1914, Poincaré 1902). Their reference-determining elements are invariant (or quasi-invariant) mathematical equations or structures. In Newtonian mechanics, the equation of motion, $\mathrm{F}_{\mathrm{i}}=\mathrm{ma}_{\mathrm{i}}=$ $\mathrm{dp}_{\mathrm{i}} / \mathrm{dt}, \mathrm{a}_{\mathrm{i}}=\mathrm{dv}_{\mathrm{i}} / \mathrm{dt}, \mathrm{v}_{\mathrm{i}}=\mathrm{dx}_{\mathrm{i}} / \mathrm{dt}(\mathrm{i}=1,2,3)$, relates the concept of a body's mass with the force that is exerted on the body. In relativistic mechanics, the corresponding equations for motion, $\mathrm{f}^{\mathrm{\mu}}=\mathrm{ma}^{\mathrm{\mu}}=\mathrm{dP}^{\mathrm{\mu}} / \mathrm{d} \tau, \mathrm{a}^{\mathrm{\mu}}=\mathrm{dU^{ \textrm { \mu } }} / \mathrm{d} \tau$, $\mathrm{U}^{\mathrm{\mu}}=\mathrm{dx}^{\mathrm{\mu}} / \mathrm{d} \tau$ ( $\mathrm{m}$ is the rest mass, $\mathrm{P}^{\mathrm{\mu}}$ is the energy-momentum 4-vector, $\mathrm{\mu}=$ 
$0,1,2,3)$, relates the concept of mass-energy with four-force. The idea of classical mass and the relativistic concept of mass-energy are all incorporated into Newtonian and relativistic mechanics. Just as classical mass is the ratio of force to acceleration in Newton's law of motion, so, too, is its relativistic counterpart the ratio of four-force to four-acceleration in the relativistic law of motion.

Just as classical mass is preserved before and after the collision of bodies, each component of mass-energy and the three-momentum vector is always preserved before and after the collision of bodies. That is, mass-energy is a quantity that is conserved and which inherits its classical counterpart. While Newtonian mechanics can explain the collision process, the emission of a photon from a body can only be explained by relativistic mechanics. In fact, the relativistic equation of motion, $\mathrm{f}^{1}=\mathrm{ma}^{\mathrm{\mu}}$, is derived from these premises: (1) the satisfaction of the relativity principle, (2) the correspondence with the relativistic law of inertia $\left(\mathrm{dU}^{\mathrm{\mu}} / \mathrm{d} \boldsymbol{\tau}=0\right.$, as $\left.\mathrm{f}^{\mathrm{r}}=0\right)$, and (3) the Newtonian limit $\left(\mathrm{f}^{1}=\mathrm{ma}^{\mathrm{\mu}}\right.$ approximates to $\mathrm{F}_{\mathrm{i}}=\mathrm{ma}_{\mathrm{i}}$, as $\mathrm{U}^{\mathrm{p}} \ll \mathrm{c}$ ) (Hartle 2003). This shows that the mass at rest and the three-momentum, respectively, inherit the concept of mass and momentum from classical mechanics. Along these lines, structural realists, such as Duhem and Poincaré, claim that, although the contents of a given theory are discarded, its underlying mathematical structures are invariant (or structurally invariant) in the course of the theory change:

There was a continuity or accumulation in the shift, but the continuity is one of form or structure, not of content. (Worrall 1987, 117)

This mathematical continuity becomes manifest from the perspective of the group structure of space-time viewpoint. The geometric properties of space-time consist of a topological Lie group, which involves the transformations of rotations, boosts and translation on $\mathbf{R}^{4}$. (see Appendix A) That is, the Lorentz transformation preserving the space-time metric can be represented as a combination of the three transformations. The concept of energy and momentum, within this framework, arise as the generators of transformations on the group of translations. Whilst this group in the relativistic case is the Lorentz group, the corresponding group in the non-relativistic case is the Galilean group. In both cases, the concept of energy, 
which is used as total relativistic mass, appears as the generator of time translations. In this context, Saunders views the relationships between the concepts of the rest mass in both physics as involving the group structure:

The non-relativistic mass, in contrast, has a quite different interpretation ..., bound up with more detailed properties of the respective Lie algebras: in the case of the [inhomogeneous Galilean group], to the 'neutral elements' of the algebra (it therefore defines the momentum and the energy in conjunction with the velocity); in the relativistic case to the Casimir invariants (a function of elements of the Lie algebra, not a separate element). In both cases these quantities have vanishing Lie bracket with every element of the Lie algebra; they are therefore conserved. One has a quite reasonable understanding of their inter-relationships as provided by the theory of group contractions. (Saunders 1993, 304)

So, by introducing the concepts of the energy-momentum 4-vector and the rest mass, the relationships between the concepts of mass can be captured. This concept unifies mass, energy and momentum without attempting to unify its physical nature. This unification is incapsulated by the common mathematical equations and group structures. One can say that the common mathematical structures, which survive scientific revolution, are in fact what theoretical terms refers to. Accordingly, Kuhn's attempt to use the concept of mass to support the occurrence of a radical conceptual change does not succeed.

\section{The case of incommensurable space-time measurements}

While the above case for the revolutionary conceptual change in the notion of mass is not supported by space-time geometry, Kuhn's other case for the incommensurability thesis as applied to the Newton-Einstein shift is concerned with an alleged conceptual change in the notion of space-time itself. In the rest of this section, I will argue that this case also in fact fails to show that the development of Einsteinian physics was revolutionary.

According to Kuhn, given that the concept of space and time provides the foundation of both Newtonian and Einsteinian physics, the change of 
these concepts generates a revolution in the conceptual network. The transition from Newtonian to Einsteinian physics can be characterized as a holistic one in the sense that it involves changes in a range of other interrelated concepts:

To make the transition to Einstein's universe, the whole conceptual web whose strands are space, time, matter, force, and so on, had to be shifted and laid down again on nature whole. (Kuhn $1962,149)$

Within Newtonian physics, simultaneities and temporal intervals between any two events are all absolute in that they are the same for all inertial observers. In other words, two events that are simultaneous for an inertial observer are simultaneous for any other inertial observer regardless of her relative motion, and the same holds for temporal intervals. Spatial intervals between two simultaneous events are also absolute. Within Einsteinian physics, on the other hand, simultaneity, and both temporal and spatial intervals between any two events are all relative to the motions of inertial observers.

Kuhn maintains that these differences between the two theoretical frameworks show that the conceptual change involved was revolutionary. Advocates of the competing paradigms, according to Kuhn, experienced a "transition of vision," which meant that the two sets of scientists observed totally different worlds:

One [set of scientists] is embedded in a flat, the other in a curved, matrix of space. Practicing in different worlds, the two groups of scientists see different things when they look from the same point in the same direction. (ibid., 150) ${ }^{3}$

First of all, we need to ask what the occurence of this "transition of vision" exactly involves in this context. In the aforementioned first quotation, Kuhn

3 This clearly separates Kuhn's revolutionary view from any evolutionary view. The latter must maintains that the realtivistic modifications of kinematic and dynamic concepts conserve or quasi-conserve essential observational and theoretical components of Newtonian physics. An evolutionary view, for example, claims the continuity of relativistic kinematic and dynamic concepts in the classical limit. 
suggests the occurrence of a change in the relationship between the fundamental conceptual elements, i.e., a structural change of the whole conceptual network. And the second quotation refers to a change of empirical substructures as a result of the conceptual change. So, given that measurements are essentially interwoven within different space-time concepts, they cannot provide a neutral basis from which to evaluate the relative empirical strengths of the two theories. Accordingly, the incommensurablity thesis involves the claim that no neutral observational basis exists due to theory ladenness.

Kuhn's conclusion is based on two premises, (1) the revolutionary conceptual change in notions of space and time, and (2) theory-ladenness of the measurements highlightened by those conceptual changes. From these two premises, Kuhn concludes that no neutral observational grounds exist for comparisons between the measurements in Newtonian and Einsteinian physics.

Two strategies might be employed to undermine Kuhn's argument. The first is to undermine one or both of the above premises. The second strategy is to argue that Kuhn's conclusion cannot be guaranteed even if we accept both premises. I will choose the second strategy. In this section, I will not attempt to undermine either the claim of the revolutionary development of the concept of space and time, or that of the theory-ladenness of the measurements based on the two different space-time theories. The strategy is instead to employ the very weapons of Kuhn's own argument. What will be argued is that even if we admit that radical conceptual changes occurred in the notions of space and time and we accept the theory-ladenness of the measurements at issue, there still exists a neutral observational basis from which to evaluate the relative evidential strengths of Newtonian and Einsteinian physics.

We need first to take a close look at the way Kuhn's premises can be understood within the context of this theory-change. As for theory-ladenness, the measurements of length are, as both sides agree, made with rigid rulers. It seems that all that the measurements with the rulers show is that if their two arms were aligned, their ends would coincide. How, then, can they be "theory-laden"?

Yet, it can in fact be argued that measurements with these instruments are dependent on a specific space-time theory, given that the length and 
interval of a rigid ruler and a clock are interpreted differently with respect to the two possible embedding theories. This is because the embedding theories are concerned with the spatio-temporal intervals between events themselves. Given that the length of two different ends of the rigid rods represents the relationships between two different spatio-temporal events, it could be differently interpreted with respect to different space-time theories. The length of a rigid ruler and the interval measured by a clock, according to Newtonian physics, are interpreted as invariant irrespective of their state of motion. On the other hand, within Einsteinian physics, the same instruments are posited as possibly experiencing length contractions due to their state of motion.

If, on the contrary, the measurements of spatio-temporal intervals employ instruments such as a light pulse and a clock, then theory-ladenness of spatial measurements occurs in the opposite way. Geroch shows how the measurement of spatio-temporal intervals is possible with light pulses and clocks (Geroch 1981, 69-72). Light pulses can be employed to probe spacetime because "light, once emitted, moves within the environment of spacetime, independently of what the emitter was doing" (ibid., 72). And by employing mirrors, "one can arrange for the light to get back to us to tell us what space-time is like" (ibid.). Observers who carry a clock with them can evaluate the spatio-temporal intervals between the observer and a specific event by evaluating the time it takes for the light sent by them to be reflected back from the event. The clocks carried by moving observers are obviously neutral between Newtonian and Einsteinian theories. Within the latter framework, the temporal intervals of the clock are distorted only when an observer measures the clocks of the others which are in motion relative to the observer. In the case of measuring the observer's own clock, its measurement results will be identical regardless of whichever theory it is based on. Yet, the light pulses are laden with a specific space-time theory here. According to the special theory of relativity, the speed of the light pulses is constant regardless of the motion of an observer relative to the light source. Yet, in Newtonian physics, the speed of light pulse is posited to change depending on the observer's motion with respect to the light pulse. Given that the dimensions of the same instruments for the measurement of spatial intervals are differently interpreted in different theories, it 
seems that the instruments by no means provide a neutral observational basis between the two theories.

Consider, for example, the Michelson-Morley experiment. As it was designed to measure the effect of the ether on the speed of light pulses, this experiment can in fact be interpreted as measuring the structure of spacetime. To detect the effect of the ether, the apparatus is equipped with an interferometer, which is posited as being at rest with respect to the earth moving through the ether. The idea of the experiment is to compare the speed of light pulses moving through the ether frame with the speed of light pulses perpendicular to the frame. By using half-silvered mirrors, light pulses are reflected to travel back and forth along two different directions, once along the direction of motion of earth and once at right angle to that motion. Although this experiment was originally designed to measure the speed of light with respect to the frame of ether, we can also employ this experiment in order to examine whether or not Lorentz contraction occurs.

The occurrence of length differences for these two distinct round-trip journeys can be employed as an appraisal of Newtonian and relativity theories. In this experiment, the existence of a length contraction of the interferometer arms is a component that plays a crucial role in producing the length difference of the two round-trip journeys, which is the key to the appraisals of Newtonian and Einsteinian physics. When this experiment is prepared, an ordinary ruler is employed to determine the lengths of the interferometer arms. We also could think of the case that the experimental design employs light pulses and clock in order to determine the lengths of the interferometer arms. In both cases, when one assumes that the ruler (or light pulses and clock) can be employed to determine the lengths of the arms, one assumes the very theory to be appraised (Laymon 1988, 250). The former case employing the ruler assumes that there does not exist Lorentz contraction effect, while the latter using light pulses assumes the opposite. Then, do the difference of the concept of space-time and the theoryladenness of space-time measurement guarantee the incommensurability Kuhn suggested?

Our answer is 'no.' Although one admits that a component of the experiment is theory-laden as mentioned, this experiment still produces a neutral observational basis for the appraisal of Newtonian and Einsteinian 
physics (Laymon 1988, Cho 1996). Consider the lengths of round-trip journeys that are experienced by (1) the light pulses moving through the ether frame and (2) the light pulses moving perpendicular to the frame. In the original experimental setting, where $\mathrm{c}$ and $\mathrm{v}$ are the velocity of light and the earth in its orbit respectively and $\mathrm{D}$ is the distance of interferometer arm length, (1) is calculated as $2 \mathrm{D} /\left(1+\mathrm{v}^{2} / \mathrm{c}^{2}\right)$, while $(2)$ is $2 \mathrm{D}\left(1+\mathrm{v}^{2} / \mathrm{c}^{2}\right)^{-1 / 2}$, which is $2 \mathrm{D}\left(1+\left(\mathrm{v}^{2} / 2 \mathrm{c}^{2}\right)\right)$ if terms higher than $(\mathrm{v} / \mathrm{c})^{2}$ are neglected. (See Appendix B) As Michelson and Morley rotated the whole apparatus through $90^{\circ}$, the predicted displacement of the interference fringe becomes $2 \mathrm{D}\left(\mathrm{v}^{2} / \mathrm{c}^{2}\right)$ (Michelson and Morley 1887, 336).

In order to consider the possibility of the Lorentz contraction of the interferometer arms, we can consider a modified analysis of the result of the experiment, which assumes that the interferometer arm lengths can vary (Silverstein 1914). In other words, the interferometer arm that is parallel to the motion through the ether frame experiences Lorentz contraction. According to Laymon, whichever theory we employ, the length of the two paths of the light travels in the two different directions, is calculated as the same, if terms higher than $(\mathrm{v} / \mathrm{c})^{2}$ are neglected (Laymon 1988). The existence or non-existence of contraction of the moving ruler by no means influences the anticipated effect of the difference of the lengths of the two paths. This result stems from the fact that the final outcome is a function of the sum of the lengths of the two interferometer arms, i.e., the lengths of arms initially parallel and the lengths of arms orthogonal to the direction of motion. Let the length of the former be $D_{h}$ and the length of the latter be $D_{v}$. Given a $90 \mathrm{o}$ rotation of the interferometer, the anticipated path length change is $\left[D_{h}+D_{v}\right]\left(v^{2} / c^{2}\right)$, which is a function of the sum of the interferometer arm lengths. When we assume the hypothesis of Lorentz contraction to decide the length of the rulers, the corrected $D_{h}$, when an ordinary ruler yields a value of 11 meters for both $D_{h}$ and $D_{v}, D_{h}$ is calculated as 11(1 $\left.\mathrm{v}^{2} / \mathrm{c}^{2}\right)^{1 / 2}$. Ignoring higher order terms, $\mathrm{D}_{\mathrm{h}}$ is obtained as $11\left(1-\left(\mathrm{v}^{2} / 2 \mathrm{c}^{2}\right)\right)$. Inserting this corrected value as an input value, the fringe shift becomes $2\left[11+11\left(1-\left(\mathrm{v}^{2} / 2 \mathrm{c}^{2}\right)\right)\right]\left(\mathrm{v}^{2} / \mathrm{c}^{2}\right)$. This then yields $2[11+11]\left(\mathrm{v}^{2} / \mathrm{c}^{2}\right)$ by expanding and ignoring terms of higher than second order. (See Appendix C)

This is the same as the anticipated measurement in the case that no contraction of the ruler is assumed. So whether or not the length contraction 
is posited, the derived result of the fringe shift is the same. From this calculation, Laymon concludes:

All of this means that while it is true that the actual length of the interferometer arms is a varying function of the very theories to be tested, when measuring that length the computational effect of assuming different theories from among the set to be tested is inconsequential. Hence, the phenomena (of fringe shift in rotating equal-arm interferometers) can be determined with an accuracy sufficient for testing the relevant theories regardless of which of the competing theories is chosen to specify the measurement procedures to be used to determine the experimental initial condition of length. (Laymon 1988, 252-53)

We can see that, although a component of the measurement depends on a specific space-time theory, the experiments, whichever theories we employ, still provide a neutral observational basis for the appraisal of Newtonian and Einsteinian physics. So, theory-ladenness of the measurements highlightened by those conceptual changes within space-time theories does not guarantee Kuhn's conclusion of the non-existence of neutral observational grounds in the comparisons between the measurements in Newtonian and Einsteinian physics.

\section{Conclusion}

It has been argued that Kuhn's incommensurability thesis is by no means supported by his own cases employing the concept of mass and spacetime measurement within Newtonian and Einsteinian physics. Kuhn claims that the conceptual change in the notion of mass supports his incommensurability thesis, and also that this thesis is supported by the non-existence of a neutral observational basis for space-time measurements, stemming from the conceptual change in the notion of space-time. Yet, as regards the first, it has been argued that the concept of "relativistic mass," which Kuhn claims to be incommensurable with its classical counterpart (classical mass), is not in fact a physically meaningful concept. Furthermore, by introducing the energy-momentum 4-vector, we can clearly see the inter-relationship 
between classical mass and its relativistic counterpart. And as regards the second case, it has been argued that although such space-time measurements are in a sense theory-laden, a neutral observational basis between Newtonian and Einsteinian physics can nonetheless really be secured. Accordingly, these two cases fail to support Kuhn's incommensurability thesis.

\section{Appendix $A$}

The concept of continuous symmetry, such as rotational symmetry in 3D Euclidean space and Lorentz symmetry in Minkowski space-time, can be captured by Lie groups. Lie groups are manifolds that form groups. A manifold is a space which resembles Euclidean space in a small neighborhood of each point. For the manifold to be a Lie group, a group multiplication between every pairs of points of the manifold can be defined.

This employment of a Lie group inherits the intuition of Felix Klein's Erlangen program, which emphasizes the formal properties of symmetry groups in characterizing differential equations and geometrical objects.

According to this program, symmetry groups determine the essence of equations and geometries, since their genuine characteristics are the invariant quantities under a group of specific transformations. For example, in Newtonian space-time, the transformations between coordinate systems representing inertial frames form Lie groups, and the symmetries of these groups distinguish the invariant geometric objects, independently of the choice of any specific reference frame. Along these lines, in Minkowski spacetime, the Lorentz transformation, which incorporates the transformations of rotations, boosts and translation on $\mathbf{R}^{4}$, forms a Lie group. That is, the Lorentz transformation preserving the space-time metric can be represented as a combination of the three transformations.

A set of group generators is a set of group elements such that repeated application of the generators on themselves and each other can produce all the group elements. At this point, one can see the connection between the existence of conserved quantities and symmetry properties of a given physical system. The concept of energy and momentum, within Newtonian framework, arise as the generators of the group of temporal and spatial translations. 
Whilst this group in the relativistic case is the Lorentz group, the corresponding group in the non-relativistic case is the Galilean group. In both cases, the concept of energy, which is used as total relativistic mass, appears as the generator of time translations.

\section{Appendix B}

As Michelson and Morley (1887) define, $\mathrm{c}$ is the velocity of light, $\mathrm{v}$ is the velocity of the earth in its orbit respectively and $\mathrm{D}$ is the distance of interferometer arm length. The calculations of the lengths of round-trip journeys that are experienced by (1) and (2) can be given as follows.

Let $\mathrm{T}=$ time that light occupies to outgoing pass, and $\mathrm{T}_{1}=$ time that light occupies to returning pass. Then, $\mathrm{T}$ is calculated as $\mathrm{D} /(\mathrm{c}+\mathrm{v})$, and $\mathrm{T}_{1}$ is calculated as $\mathrm{D} /(\mathrm{c}+\mathrm{v})$. And the whole time of the round-trip is $\mathrm{T}+$ $\mathrm{T} 1=\mathrm{D} /(\mathrm{c}-\mathrm{v})+\mathrm{D} /(\mathrm{c}+\mathrm{v})=2 \mathrm{Dc} /\left(\mathrm{c}^{2}-\mathrm{v}^{2}\right)=2 \mathrm{Dc} /\left[\mathrm{c}^{2}\left(1-\mathrm{v}^{2} / \mathrm{c}^{2}\right)\right] \sim[2 \mathrm{D}(1$ $\left.\left.+\mathrm{v}^{2} / \mathrm{c}^{2}\right)\right] / \mathrm{c}$, if terms higher than $(\mathrm{v} / \mathrm{c})^{2}$ is neglected. So, the length of roundtrip journeys, i.e., (1), is $\mathrm{c}\left(\mathrm{T}+\mathrm{T}_{1}\right)=2 \mathrm{D}\left(1+\mathrm{v}^{2} / \mathrm{c}^{2}\right)$.

The calculation of the time of the round-trip of other path is similar as the derivation of the time dilation employing a light pulse. The travel time of the round-trip of a light pulse is $2 \mathrm{D} /\left(\mathrm{c}^{2}-\mathrm{v}^{2}\right)^{1 / 2}=2 \mathrm{D} /\left[\mathrm{c}^{2}\left(1-\mathrm{v}^{2} / \mathrm{c}^{2}\right)\right]^{1 / 2}=$ $\left[2 \mathrm{D}\left(1-\mathrm{v}^{2} / \mathrm{c}^{2}\right)^{-1 / 2}\right] / \mathrm{c}$, which is $\left[2 \mathrm{D}\left(1-\left(\mathrm{v}^{2} / 2 \mathrm{c}^{2}\right)\right)\right] / \mathrm{c}$ if terms higher than $(\mathrm{v} / \mathrm{c})^{2}$ is neglected. Accordingly, its length, i.e., (2), is $2 \mathrm{D}\left(1+\left(\mathrm{v}^{2} / 2 \mathrm{c}^{2}\right)\right)$.

\section{Appendix $C$}

Lorentz constructed his ether theory which explains the undetectability of the relative motion of ether with respect to earth. According to this theory, the frame of ether can be distinguished as Newton's absolute space. And the length contraction of a body in line with its motion explains the null result of the Michelson-Morley experiment.

A central idea of Lorentz ether theory is summarized as the "theorem of corresponding states". The theorem says that the laws of electromagnetics are invariant irrespective of the choice of the reference frame, i.e., whether it is measured with respect to the stationary ether frame or the moving one.

The length contraction of a moving body in its moving direction enables physicists to identify the "preferred" reference frame based on Lorentz's 
ether. The formula of the length contraction is given as $\mathrm{l}=\mathrm{l}_{\mathrm{o}}\left(1-\mathrm{v}^{2} / \mathrm{c}^{2}\right)^{1 / 2}$ $\sim \mathrm{l}_{\mathrm{o}}\left(1-\left(\mathrm{v}^{2} / 2 \mathrm{c}^{2}\right)\right)$, where lo is the length with respect to the stationary ether.

In the Michelson-Morley experiment, the distance of interferometer arm length is set to $\mathrm{D}$ whether it is measured in its moving direction or its perpendicular one. Then, the predicted displacement of the interference fringe is calculated as $2 \mathrm{D}\left(\mathrm{v}^{2} / \mathrm{c}^{2}\right)$. (see Appendix 1) In contrast, under the assumption that the interferometer arm lengths can vary in accordance with Lorentz ether theory, the predicted displacement of the interference fringe is calculated as $2\left(D_{\mathrm{h}}+D_{\mathrm{v}}\right)\left(\mathrm{v}^{2} / \mathrm{c}^{2}\right)$.

Since $\mathrm{Dh}$ is what Lorentz contraction is applied to, $\mathrm{D}_{\mathrm{h}}$ is calculated as $11\left(1-\mathrm{v}^{2} / \mathrm{c}^{2}\right)^{1 / 2} \sim 11\left(1-\left(\mathrm{v}^{2} / 2 \mathrm{c}^{2}\right)\right)$. ( $\mathrm{D}$ h with respect to the stationary ether is measured as 11 meters, as Michelson and Morley prepared.) Inserting this corrected value as an input value, the predicted displacement of the interference fringe becomes $2\left[11+11\left(1-\left(\mathrm{v}^{2} / 2 \mathrm{c}^{2}\right)\right)\right]\left(\mathrm{v}^{2} / \mathrm{c}^{2}\right)=2\left[11+11-11\left(\mathrm{v}^{2} / 2 \mathrm{c}^{2}\right)\right]$ $\left(\mathrm{v}^{2} / \mathrm{c}^{2}\right)$. This then yields $2[11+11]\left(\mathrm{v}^{2} / \mathrm{c}^{2}\right)$ by ignoring terms of $\left(\mathrm{v}^{2} / \mathrm{c}^{2}\right)^{2}$.

\section{References}

Bird, Alexander. 2000. Thomas Kuhn. Princeton: Princeton University Press. Born, Max. 1965. My Life and My Views. New York: Charles Scribner's Sons. Cho, Inrae. 1996. "The Methodological Challenge of Kuhn's Incommensurability Thesis." Philosophy, Korea Philosophical Association 46: 155-88.

Cohen, I. Bernard. 1985. Revolution in Science. Cambridge MA: Harvard University Press.

Duhem, Pierre. 1914. The Aim and Structure of Physical Theory. Princeton: Princeton University Press.

Einstein, Albert. 1905. "On the Electrodynamics of Moving Bodies." In Principle of Relativity, edited by Einstein, Albert., Hendrik A. Lorentz, Hermann Minkowski, and Hermann Weyl. 1952. Translated by W. Perrett and G.B. Jeffery, 35-65. New York: Dover Books.

Einstein, Albert. 1919. "What is the Theory of Relativity?" In Ideas and Opinions. 1982. 227-32. New York: Bonanza Books.

Einstein, Albert. 1934. Essay in Science. New York: Philosophical Library

Einstein, Albert. 1949. "Autobiographical notes." In Albert Einstein, Philosopher-

Scientist, edited by Paul Arther Schilpp. Chicago: Open Court.

Einstein, Albert. 1965. My Life and My Views. New York: Charles Scribner's Sons. 
Feyerabend, Paul. K. 1962. "Explanation, Reduction, and Empiricism." In Minnesota Studies in the Philosophy of Science, vol III, edited by H. Feigl and G. Maxwell, 28-97. Dordrecht: D. Reidel Publishing Company.

Field, Harty. 1973. "Theory Change and The Indeterminacy of Reference." The Journal of Philosophy 70(14): 462-81. https://doi.org/10.2307/2025110

Geroch, Robert. 1978. Relativity from A to B. Chicago: University of Chicago Press.

Hardin, Clyde L. and Alexander Rosenberg. 1982. "In Defence of Convergent Realism." Philosophy of Science 49(4): 604-15. https://doi.org/10.1086/289080

Hartle, James B. 2003. Gravity: An Introduction to Einstein's General Relativity. New York: Pearson

Jammer, Max. 2000. Concepts of Mass in Contemporary Physics and Philosophy. Princeton: Princeton University Press.

Klein, Martin J. 1975. "Einstein on Scientific Revolutions," Vistas in Astronomy 17: 113-33. https://doi.org/10.1016/0083-6656(75)90053-7

Kitcher, Phillip. 1993. The Advancement of Science, New York: Oxford University Press.

Kuhn, Thomas I. 1962. The Structure of Scientific Revolutions. Chicago: University of Chicago Press.

Lakatos, Imre and Alan Musgrave. Eds. 1970. Criticism and the Growth of Knowledge, London: Cambridge University Press.

Laymon, Ronald. 1988. "The Michelson-Morley Experiment and the Appraisal of Theories." In Scrutinizing Science, edited by Donovan, Arthur, Larry Laudan, and Rachel Lauda., 245-66. Dordrecht: Kluwer. https://doi.org/10.1007/97894-009-2855-8_12

Michelson, Albert A. and Morley. Edward W. 1887. "On the Relative Motion of the Earth and the Luminiferous Ether," American Journal of Science 34: 33345. https://doi.org/10.2475/ajs.s3-34.203.333

Morrison, Margaret, 2000. Unifying Scientific Theories: Physical Concepts and Mathematical Structures. Cambridge: Cambridge University Press.

Oas, Gary. 2006. "On the Abuse and Use of Relativistic Mass." http://arxiv.org/abs/physics/0504110

Okun, Lev. B. 1989. "The Concept of Mass," Physics Today 42(6): 31-36. https://doi.org/10.1063/1.881171

Penrose, Roger. 2004. The Road to Reality: A Complete Guide to the Laws of the Universe. Knopf.

Poincaré, Henri. 1902, Science and hypothesis, New York: Dover.

Popper, Karl. 1959. The Logic of Scientific Discovery. London: Hutchinson. Resnik, Robert. 1968. Introduction to Special Relativity. New York: Wiley. 
Saunders, Simon. 1993. "To What Physics Corresponds." In Correspondence, Invariance, and Heuristics, Essays in Honour of Heinz Post, 295-25, Dordrecht, Reidel. https://doi.org/10.1007/978-94-017-1185-2_15

Silberstein, Ludwik. 1914. The Theory of Relativity. London: London, Macmillan. Taylor, Edward. F. and John. Archibald Wheeler. 1966. Spacetime Physics. San Francisco: W. H. Freeman.

Taylor, Edward. F. and John. Archibald Wheeler. 1992. Spacetime Physics 2nd edition. San Francisco: W. H. Freeman.

Withrow, Gerald James. ed. 1967. Einstein: The Man and His Achievement. A series of broadcast talk. London: BBC.

Worrall, John. 1989. "Structural Realism: The Best of Both Worlds?" Dialetica 43: 99-124. https://doi.org/10.1111/j.1746-8361.1989.tb00933.x 